\title{
Derivation of dynamical integral inequalities based on two-dimensional time scales theory
}

\author{
Zareen A. Khan ${ }^{1}$, Fahd Jarad ${ }^{2 *}$ (D) Aziz Khan ${ }^{3 *}$ and Hasib Khan ${ }^{4}$
}

\section{"Correspondence:}

fahd@cankaya.edu.tr;

azizkhan927@yahoo.com

${ }^{2}$ Department of Mathematics,

Çankaya University, Ankara, Turkey

${ }^{3}$ Department of Mathematics and

General Sciences, Prince Sultan

University, P.O. Box 66833, Riyadh,

11586, Saudi Arabia

Full list of author information is

available at the end of the article

\begin{abstract}
The main goal of this paper is to set up some new estimates of a specific class of dynamic integral inequalities (DII) which are partially linear on a time scale $\mathbb{T}$ with two independent variables. These, from the one hand, sum up and, on the other hand, offer a helpful method for both the qualitative and quantitative study of dynamic equations on time scales. Some applications are taken into consideration to show the validity of the fundamental results.
\end{abstract}

MSC: 26D10; 26D15; 26E70

Keywords: Integral inequalities; Time scales; Nondecreasing; Dynamic equations

\section{Introduction}

Differential and integral inequalities have ended up being valuable instruments in investigating the differential and integral equations that are constructed by numerous analysts (see [1-17]). The analysis of the theory of time-scale dynamic equations, which goes back to its author Hilger [18], is a recent field of mathematics that has gained much interest. The trendy thought is to demonstrate an equation for a dynamic circumstance or a dynamic inequality wherein the area of the unknown characteristic is a presumed time scale $\mathbb{T}$. The justification for considering time scales is to unify continuous and discrete inspection. Among diverse aspects of the concept, we observe that dynamic inequalities increase and unify different views of both difference and differential equations in an anticipated mode; see [19-21] and the references therein. Pachpatte [22] initially unifies the existing fundamental inequality

$$
x(r) \leq b(r)+\int_{r_{0}}^{r} h(u) x(u) \Delta u, \quad r \in \mathbb{T},
$$

provided with $x, h$ being right-dense continuous functions, $x \geq 0$.

After that in 2009, Li [23] achieved an integral inequality of the form

$$
x(r) \leq x_{0}+\int_{r_{0}}^{r}[h(u) x(u)+m(u)] \Delta u+\int_{r_{0}}^{r} g(u)[z(u, l) x(l) \Delta l] \Delta u,
$$

(c) The Author(s) 2020. This article is licensed under a Creative Commons Attribution 4.0 International License, which permits use, sharing, adaptation, distribution and reproduction in any medium or format, as long as you give appropriate credit to the original author(s) and the source, provide a link to the Creative Commons licence, and indicate if changes were made. The images or other third party material in this article are included in the article's Creative Commons licence, unless indicated otherwise in a credit line to the material. If material is not included in the article's Creative Commons licence and your intended use is not permitted by statutory regulation or exceeds the permitted use, you will need to obtain permission directly from the copyright holder. To view a copy of this licence, visit http://creativecommons.org/licenses/by/4.0/. 
where $z(u, l) \geq 0, z^{\Delta}(u, l) \geq 0$ for $r, l \in \mathbb{T}$ and $l \leq r$. Recently, Sun and Hassan [24] discovered a nonlinear integral inequality related to time scales given by

$$
x(r) \leq b(r)+j(r) \int_{r_{0}}^{r}\left[h(u) x(u)+g(u) x^{\xi}(\sigma(u))-z(u) x^{\psi}(\sigma(u))\right] \Delta u,
$$

where $b, j, h, g, z: \mathbb{T}^{\kappa} \rightarrow \mathbb{R}_{+}$are rd-continuous functions and $\xi, \psi$ are positive constants with $0<\xi<1<\psi$.

During the last couple of years, many researchers have proved a few integral inequalities utilizing dynamic time scale equations [25-29]. In light of the above work, we proceed with our examination to acquire some new two-dimensional integral inequalities that are partially linear on time scales. The obtained results additionally supply a reachable device for the look at qualitative properties of solutions of integral and dynamic equations.

The rest of the paper is composed as follows. In Sect. 2, we describe principal relations and tentative lemmas that are strategic guides for our principle results. The hypothetical discussions with finishing up corollaries and remarks are gathered in Sect. 3. The last section is considered to delineate the uses of the theoretical investigations.

\section{Essentials and lemmas on time scales}

Without loss of generality, at some point of in this work, let time scales $T^{*}$ and $T^{\diamond}$ with delta differentiation operators $\sigma_{1}, \sigma_{2}$ and $\Delta_{1}, \Delta_{2}$ respectively, contain at least two points $\zeta_{0}, \xi \in T^{*}, r_{0}, \psi \in T^{\diamond}, \xi>\zeta_{0}, \psi>r_{0}, T_{1}^{*}=\left[\zeta_{0}, \infty\right) \cap T^{*}, T_{1}^{\diamond}=\left[r_{0}, \infty\right) \cap T^{\diamond}, \Upsilon=T^{*} \times T^{\diamond}$; $x^{\Delta_{1}}(\zeta, r), x^{\Delta_{2}}(\zeta, r)$, and $x^{\Delta_{1} \Delta_{2}}(\zeta, r)=x^{\Delta_{2} \Delta_{1}}(\zeta, r)$ are the delta derivatives of a function $x(\zeta, r)$ with respect to $\zeta, r$, and $p r$, respectively, for $\zeta, r \in \mathbb{R} ; \mathbb{R}$ stands for the set of real numbers, $\mathbb{R}_{+}=[0, \infty), j_{0} \in \mathbb{T}_{k}, \mathbb{T} \in \mathbb{T}^{k}, \mathbb{T}>\zeta_{0}, C_{r d}$ is the set of all rd-continuous functions, $\Re$ defines the set of all regressive and rd-continuous functions and $\Re^{+}=\{x \in \Re$ : $1+\varsigma(\zeta) x(\zeta)>0, \zeta \in \mathbb{T}\},(x \oplus c)(\zeta):=x(\zeta)+c(\zeta)+\varsigma(\zeta) x(\zeta) c(\zeta)$ for all $\zeta \in \mathbb{T}^{k}$. On time scales, the user is supposed to be comfortable with the theory and basic ideas regarding the analytics. The monograph [30] is recommended for further details of time scale ideology.

Some important lemmas in this paper are as follows:

Lemma 2.1 ([23]) Let $\zeta_{0} \in \mathbb{T}^{k}$ and $\Pi: \mathbb{T} \times \mathbb{T}^{k} \rightarrow \mathbb{R}$ be continuous at $(\zeta, \zeta)$, where $\zeta>\zeta_{0}$ and $\zeta \in \mathbb{T}^{k}$. Assume that $\Pi^{\Delta}(\zeta, \cdot)$ is rd-continuous on $\left[\zeta_{0}, \sigma(\zeta)\right]_{\mathbb{T}}$ and for every $\epsilon>0, a$ neighborhood $\Sigma^{*}$ of $\zeta$, independent of $a \in\left[\zeta_{0}, \sigma(\zeta)\right]_{\mathbb{T}}$, such that

$$
\left|[\Pi(\sigma(\zeta), \eta)-\Pi(s, a)]-\Pi^{\Delta}(\zeta, a)[\sigma(\zeta)-s]\right| \leq \epsilon|\sigma(\zeta)-s|, \quad s \in \Sigma^{*}
$$

where $\Pi^{\Delta}$ symbolizes the derivative of $\Pi$ w.r.t. the first variable, and so

$$
x(\zeta)=\int_{\zeta 0}^{\zeta} \Pi(\zeta, a) \Delta a
$$

yields

$$
x^{\Delta}(\zeta)=\int_{\zeta_{0}}^{\zeta} \Pi^{\Delta}(\zeta, a) \Delta a+\Pi(\sigma(\zeta), \zeta)
$$


Lemma 2.2 ([31]) Let $z^{*}>0, c^{*}>0, \xi>0, \psi>0$ be given, then for each $x \geq 0$,

$$
z x^{\xi}-c x^{\psi} \leq \frac{z(\psi-\xi)}{(\psi-1)}\left(\frac{(\psi-1) c}{(\xi-1) z}\right)^{\frac{\xi-1}{\xi-\psi}} x
$$

holds for the cases $1<\xi<\psi$ or $0<\psi<\xi<1$.

Lemma 2.3 ([32]) Suppose $x, z \in C_{r d}, c \in \mathbb{R}_{+}$. Then

$$
x^{\Delta}(\zeta) \leq z(\zeta) x(\zeta)+c(\zeta), \quad \zeta \in \mathbb{T},
$$

implies

$$
x(\zeta) \leq x\left(\zeta_{0}\right) e_{z}\left(\zeta, \zeta_{0}\right)+\int_{\zeta_{0}}^{\zeta} e_{z}(\zeta, \sigma(a)) c(a) \Delta a, \quad \zeta \in \mathbb{T}
$$

Lemma 2.4 ([22]) Assume that $x, z, c \in C_{r d}\left(\left[\zeta_{0}, \infty\right)_{\mathbb{T}}, \mathbb{R}_{+}\right)$, where $z$ is nondecreasing and not identically zero. If

$$
x(\zeta) \leq z(\zeta)+\int_{\zeta_{0}}^{\zeta} c(s) x(s) \Delta s, \quad \zeta \in\left[\zeta_{0}, \infty\right)_{\mathbb{T}},
$$

then

$$
x(\zeta) \leq z(j) e_{c}\left(\zeta, \zeta_{0}\right), \quad \zeta \in\left[r_{0}, \infty\right)_{\mathbb{T}} .
$$

\section{Dynamic integral inequalities (DII) in two dimensions}

Now we state and demonstrate our primary results:

Theorem 3.1 Suppose $x, y, l, t, u, m \in C_{r d}\left(\Upsilon, \mathbb{R}_{+}\right), z, c \in C_{r d}(\Upsilon,(0, \infty))$, y is nondecreasing and not identically zero, $\xi, \psi$ are positive constants, $1<\xi<\psi$ or $0<\psi<\xi<1$. Moreover, $m \in C_{r d}\left(\Upsilon, \mathbb{R}_{+}\right)$is nondecreasing in $\zeta, \Pi$ is defined as in Lemma 2.1 such that $\Pi^{\Delta_{1}}(\zeta, r, o, \varrho) \geq 0, \Pi^{\Delta_{2} \Delta_{1}}(\zeta, r, o, \varrho) \geq 0$ for $\zeta \geq o, r \geq \varrho$ and

$$
\begin{aligned}
x(\zeta, r) \leq & y(\zeta, r)+\int_{\zeta_{0}}^{\zeta} \int_{r_{0}}^{r} l(o, \varrho) x(o, \varrho) \Delta \varrho \Delta o \\
& +t(\zeta, r) \int_{\zeta_{0}}^{\zeta} \int_{r_{0}}^{r} \Pi(\zeta, r, o, \varrho)\left[u(o, \varrho) x(o, \varrho)+z(o, \varrho) x^{\xi}(\sigma(o), \varrho)\right. \\
& \left.-c(o, \varrho) x^{\psi}(\sigma(o), \varrho)\right] \Delta \varrho \Delta o \\
& +\int_{\zeta_{0}}^{S} m(o, r) x(o, r) \Delta o, \quad(\zeta, r) \in \Upsilon
\end{aligned}
$$

provided with

$$
\mu(\zeta, r) \Theta(\zeta, r)<1, \quad \int_{\zeta 0}^{S} m(o, r) e_{V \oplus W}\left(o, \zeta_{0}\right) \Delta o<1 .
$$


Then

$$
\begin{aligned}
x(\zeta, r) \leq & y(\zeta, r)+\frac{\int_{\zeta_{0}}^{S} m(o, r)\left[y(o, r)+\int_{\zeta_{0}}^{o} e_{V \oplus W}(o, \sigma(\tau)) D(\tau, r) \Delta \tau\right] \Delta o}{1-\int_{\zeta_{0}}^{S} m(o, r) e_{V \oplus W}\left(o, \zeta_{0}\right) \Delta o} e_{V \oplus W}\left(\zeta, \zeta_{0}\right) \\
& +\int_{\zeta_{0}}^{S} m(o, r) e_{V \oplus W}(\tau, \sigma(o)) \Delta o,
\end{aligned}
$$

where

$$
\begin{aligned}
& \Theta(\zeta, r)=t^{\Delta_{1}}(\zeta, r) \int_{\zeta_{0}}^{\sigma(\zeta)} \int_{r_{0}}^{r} \Pi(\sigma(\zeta), r, o, \varrho)(u(o, \varrho)+H(o, \varrho)) \Delta \varrho \Delta o \\
& +t(\zeta, r) \int_{\zeta_{0}}^{\zeta} \int_{r_{0}}^{r} \Pi^{\Delta_{2} \Delta_{1}}(\zeta, r, o, \varrho) H(o, \varrho) \Delta \varrho \Delta o \\
& +\int_{r_{0}}^{r} \Pi^{\Delta_{1}}(\sigma(\zeta), r, \zeta, \varrho) H(\zeta, \varrho) \Delta \varrho \\
& W(\zeta, r)=\int_{r_{0}}^{r} l(\zeta, \varrho) \Delta \varrho+t(\zeta, r) \int_{\zeta 0}^{\zeta} \int_{r_{0}}^{r} \Pi^{\Delta_{2} \Delta_{1}}(\zeta, r, o, \varrho) u(o, \varrho) \Delta \varrho \Delta o \\
& +\int_{r_{0}}^{r} \Pi^{\Delta_{1}}(\sigma(\zeta), r, \zeta, \varrho) u(\zeta, \varrho) \Delta \varrho \\
& \eta(\zeta, r)=\int_{r_{0}}^{r} l(\zeta, \varrho) y(\zeta, \varrho) \Delta \varrho+t^{\Delta_{1}}(\zeta, r) \int_{\zeta_{0}}^{\sigma(j)} \int_{r_{0}}^{r} \Pi(\sigma(\zeta), r, o, \varrho)(u(o, \varrho) y(o, \varrho) \\
& +H(o, \varrho) y(\sigma(o), \varrho)) \Delta \varrho \Delta o \\
& +t(\zeta, r) \int_{\zeta_{0}}^{\zeta} \int_{r_{0}}^{r} \Pi^{\Delta_{2} \Delta_{1}}(\zeta, r, o, \varrho)(u(o, \varrho) y(o, \varrho)+H(o, \varrho) y(\sigma(o), \varrho)) \Delta \varrho \Delta o \\
& +\int_{r_{0}}^{r} \Pi^{\Delta_{1}}(\sigma(\zeta), r, \zeta, \varrho)(u(\zeta, \varrho) y(\zeta, \varrho)+H(\zeta, \varrho) y(\sigma(\zeta), \varrho)) \Delta \varrho \\
& H(\zeta, r)=\frac{z(\zeta, r)(\psi-\xi)}{(\psi-1)}\left(\frac{(\psi-1) c(\zeta, r)}{(\xi-1) z(\zeta, r)}\right)^{\frac{\xi-1}{\xi-\psi}} \\
& V(\zeta, r)=\frac{\Theta(\zeta, r)}{1-\mu(\zeta, r) \Theta(\zeta, r)}, D(\zeta, r)=(1+\mu(\zeta, r) V(\zeta, r)) \eta(\zeta, r)
\end{aligned}
$$

Proof Employing Lemma 2.2 in (1), we get

$$
\begin{aligned}
x(\zeta, r) \leq & y(\zeta, r)+\int_{\zeta_{0}}^{\zeta} \int_{r_{0}}^{r} l(o, \varrho) x(o, \varrho) \Delta \varrho \Delta o \\
& +t(\zeta, r) \int_{\zeta_{0}}^{\zeta} \int_{r_{0}}^{r} \Pi(\zeta, r, o, \varrho)[u(o, \varrho) x(o, \varrho)+H(o, \varrho) x(\sigma(o), \varrho)] \Delta \varrho \Delta o \\
& +\int_{\zeta_{0}}^{S} m(o, r) x(o, r) \Delta o, \\
\leq & y(\zeta, r)+A(\zeta, r),
\end{aligned}
$$


where $H(\zeta, r)$ is as in (7) and a nondecreasing function $A(\zeta, r)$ is defined by

$$
\begin{aligned}
A(\zeta, r)= & \int_{\zeta_{0}}^{\zeta} \int_{r_{0}}^{r} l(o, \varrho) x(o, \varrho) \Delta \varrho \Delta o \\
& +t(\zeta, r) \int_{\zeta_{0}}^{\zeta} \int_{r_{0}}^{r} \Pi(\zeta, r, o, \varrho)[u(o, \varrho) x(o, \varrho)+H(o, \varrho) x(\sigma(o), \varrho)] \Delta \varrho \Delta o \\
& +\int_{\zeta_{0}}^{S} m(o, r) x(o, r) \Delta o
\end{aligned}
$$

and

$$
A\left(\zeta_{0}, r\right)=\int_{\zeta_{0}}^{S} m(o, r) x(o, r) \Delta o
$$

From Lemma 2.1 and by using the delta derivative (10) with respect to $j$, we deduce

$$
\begin{aligned}
& A^{\Delta_{1}}(\zeta, r) \\
& \quad \leq \int_{r_{0}}^{r} l(\zeta, \varrho) x(\zeta, \varrho) \Delta \varrho \\
& \quad+t^{\Delta_{1}}(\zeta, r) \int_{\zeta_{0}}^{\sigma(\zeta)} \int_{r_{0}}^{r} \Pi(\sigma(\zeta), r, o, \varrho)[u(o, \varrho) x(o, \varrho)+H(o, \varrho) x(\sigma(o), \varrho)] \Delta \varrho \Delta a \\
& \quad+t(\zeta, r) \int_{\zeta_{0}}^{\zeta} \int_{r_{0}}^{r} \Pi^{\Delta_{2} \Delta_{1}}(\zeta, r, o, \varrho)[u(o, \varrho) x(o, \varrho)+H(o, \varrho) x(\sigma(o), \varrho)] \Delta \varrho \Delta a \\
& \quad+t(\zeta, r) \int_{r_{0}}^{r} \Pi^{\Delta_{1}}(\sigma(\zeta), r, \zeta, \varrho)[u(\zeta, \varrho) x(\zeta, \varrho)+H(\zeta, \varrho) x(\sigma(\zeta), \varrho)] \Delta \varrho,
\end{aligned}
$$

where, using (9) in the last inequality, we get

$$
\begin{aligned}
A^{\Delta_{1}}(\zeta, r) \leq & \int_{r_{0}}^{r} l(\zeta, \varrho)(y(\zeta, \varrho)+A(\zeta, \varrho)) \Delta v \\
& +t^{\Delta_{1}}(\zeta, r) \int_{\zeta_{0}}^{\sigma(\zeta)} \int_{r_{0}}^{r} \Pi(\sigma(\zeta), r, o, \varrho)[u(o, \varrho)(y(o, \varrho)+A(o, \varrho)) \\
& +H(o, \varrho)(y(\sigma(o), \varrho)+A(\sigma(o), \varrho))] \Delta \varrho \Delta o \\
& +t(\zeta, r) \int_{\zeta 0}^{\zeta} \int_{r_{0}}^{r} \Pi^{\Delta_{2} \Delta_{1}}(\zeta, r, o, \varrho)[u(o, \varrho)(y(o, \varrho)+A(o, \varrho)) \\
& +H(o, \varrho)(y(\sigma(o), \varrho)+A(\sigma(o), \varrho))] \Delta \varrho \Delta o \\
& +t(\zeta, r) \int_{r_{0}}^{r} \Pi^{\Delta_{1}}(\sigma(\zeta), r, \zeta, \varrho)[u(\zeta, \varrho)(y(\zeta, \varrho)+A(\zeta, \varrho)) \\
& +H(\zeta, \varrho)(y(\sigma(\zeta), \varrho)+A(\sigma(\zeta), \varrho))] \Delta \varrho,
\end{aligned}
$$

so that

$$
A^{\Delta_{1}}(\zeta, r)
$$




$$
\begin{aligned}
\leq & \int_{r_{0}}^{r} l(\zeta, \varrho) y(\zeta, \varrho) \Delta \varrho+\int_{r_{0}}^{r} l(\zeta, \varrho) A(\zeta, \varrho) \Delta \varrho \\
& +t^{\Delta_{1}}(\zeta, r) \int_{\zeta_{0}}^{\sigma(\zeta)} \int_{r_{0}}^{r} \Pi(\sigma(\zeta), r, o, \varrho)[u(o, \varrho) y(o, \varrho)+H(o, \varrho) y(\sigma(o), \varrho)] \Delta \varrho \Delta o \\
& +A(\sigma(\zeta), r)\left[t^{\Delta_{1}}(\zeta, r) \int_{\zeta 0}^{\sigma(\zeta)} \int_{r_{0}}^{r} \Pi(\sigma(\zeta), r, o, \varrho)(u(o, \varrho)+H(o, \varrho)) \Delta \varrho \Delta o\right] \\
& +t(\zeta, r) \int_{\zeta_{0}}^{\zeta} \int_{r_{0}}^{r} \Pi^{\Delta_{2} \Delta_{1}}(p, r, o, v)[u(o, \varrho) y(o, \varrho)+H(o, \varrho) y(\sigma(o), \varrho)] \Delta \varrho \Delta o \\
& +t(\zeta, r) \int_{\zeta_{0}}^{\zeta} \int_{r_{0}}^{r} \Pi^{\Delta_{2} \Delta_{1}}(\zeta, r, o, \varrho) u(o, \varrho) A(o, \varrho) \Delta \varrho \Delta o \\
& +t(\zeta, r) \int_{\zeta_{0}}^{\zeta} \int_{r_{0}}^{r} \Pi^{\Delta_{2} \Delta_{1}}(\zeta, r, o, \varrho) H(o, \varrho) A(\sigma(o), \varrho) \Delta \varrho \Delta o \\
& +t(\zeta, r) \int_{r_{0}}^{r} \Pi^{\Delta_{1}}(\sigma(\zeta), r, \zeta, \varrho)[u(\zeta, \varrho) y(\zeta, \varrho)+H(\zeta, \varrho) y(\sigma(\zeta), \varrho)] \Delta \varrho \\
& +t(\zeta, r) \int_{r_{0}}^{r} \Pi^{\Delta_{1}}(\sigma(\zeta), r, \zeta, \varrho) u(\zeta, \varrho) A(\zeta, \varrho) \Delta \varrho \\
& +t(\zeta, r) \int_{r_{0}}^{r} \Pi^{\Delta_{1}}(\sigma(\zeta), r, \zeta, \varrho) H(\zeta, \varrho) A(\sigma(\zeta), \varrho) \Delta \varrho,
\end{aligned}
$$

which leads to

$$
\begin{aligned}
& A^{\Delta_{1}}(\zeta, r)=\left[t^{\Delta_{1}}(\zeta, r) \int_{\zeta_{0}}^{\sigma(\zeta)} \int_{r_{0}}^{r} \Pi(\sigma(\zeta), r, o, \varrho)(u(o, \varrho)+H(o, \varrho)) \Delta \varrho \Delta o\right. \\
& \quad+t(\zeta, r) \int_{\zeta 0}^{\zeta} \int_{r_{0}}^{r} \Pi^{\Delta_{2} \Delta_{1}}(\zeta, r, o, \varrho) H(o, \varrho) \Delta \varrho \Delta o \\
&\left.\quad+t(\zeta, r) \int_{r_{0}}^{r} \Pi^{\Delta_{1}}(\sigma(\zeta), r, \zeta, \varrho) H(\zeta, \varrho) \Delta \varrho\right] A(\sigma(\zeta), \varrho) \\
& \quad+\left[\int_{r_{0}}^{r} l(\zeta, \varrho) \Delta \varrho+t(\zeta, r) \int_{\zeta 0}^{\zeta} \int_{r_{0}}^{r} \Pi^{\Delta_{2} \Delta_{1}}(\zeta, r, o, \varrho) u(o, \varrho) \Delta \varrho \Delta o\right. \\
&\left.\quad+t(\zeta, r) \int_{r_{0}}^{r} \Pi^{\Delta_{1}}(\sigma(\zeta), r, \zeta, \varrho) u(\zeta, \varrho) \Delta \varrho\right] A(\zeta, r)+\int_{r_{0}}^{r} l(\zeta, \varrho) y(\zeta, \varrho) \Delta \varrho \\
&+t^{\Delta_{1}}(\zeta, r) \int_{\zeta_{0}}^{\sigma(\zeta)} \int_{r_{0}}^{r} \Pi(\sigma(\zeta), r, o, \varrho)[u(o, \varrho) y(o, \varrho)+H(o, \varrho) y(\sigma(o), \varrho)] \Delta \varrho \Delta o \\
&+ \\
&+t(\zeta, r) \int_{\zeta_{0}}^{\zeta} \int_{r_{0}}^{r} \Pi^{\Delta_{2} \Delta_{1}}(\zeta, r, o, \varrho)[u(o, \varrho) y(o, \varrho)+H(o, \varrho) y(\sigma(o), \varrho)] \Delta \varrho \Delta o \\
&+t(\zeta, r) \int_{r_{0}}^{r} \Pi^{\Delta_{1}}(\sigma(\zeta), r, \zeta, \varrho)[u(\zeta, \varrho) y(\zeta, \varrho)+H(\zeta, \varrho) y(\sigma(\zeta), \varrho)] \Delta \varrho \\
& \leq \Theta(\zeta, r) A(\sigma(\zeta), r)+W(\zeta, r) A(\zeta, r)+\eta(\zeta, r),
\end{aligned}
$$


with $\Theta(\zeta, r), W(\zeta, r), \eta(\zeta, r)$ defined as in (4), (5), and (6). From (8), we obtain

$$
\Theta(\zeta, r)=\frac{V(\zeta, r)}{1+\mu(\zeta, r) V(\zeta, r)}
$$

In view of (12) and (13), we obtain

$$
\begin{aligned}
A^{\Delta_{1}}(\zeta, r) & \leq \Theta(\zeta, r)\left(A(\zeta, r)+\mu(\zeta, r) A^{\Delta_{1}}(\zeta, r)\right)+W(\zeta, r) A(\zeta, r)+\eta(\zeta, r) \\
& \leq \frac{V(\zeta, r)}{1+\mu(\zeta, r) V(\zeta, r)}\left(A(\zeta, r)+\mu(\zeta, r) A^{\Delta_{1}}(\zeta, r)\right)+W(\zeta, r) A(\zeta, r)+\eta(\zeta, r)
\end{aligned}
$$

which yields

$$
\frac{1}{1+\mu(\zeta, r) V(\zeta, r)} A^{\Delta_{1}}(\zeta, r) \leq \frac{V(\zeta, r)+W(\zeta, r)+\mu(\zeta, r) V(\zeta, r) W(\zeta, r)}{1+\mu(\zeta, r) V(\zeta, r)} A(\zeta, r)+\eta(\zeta, r),
$$

or

$$
\begin{aligned}
A^{\Delta_{1}}(\zeta, r) \leq & (V(\zeta, r)+W(\zeta, r)+\mu(\zeta, r) V(\zeta, r) W(\zeta, r)) A(\zeta, r) \\
& +(1+\mu(\zeta, r) V(\zeta, r)) \eta(\zeta, r) \\
\leq & (V \oplus W)(\zeta, r) A(\zeta, r)+D(\zeta, r) .
\end{aligned}
$$

Here $D(\zeta, r)$ is as in (8). As we know that $A(\zeta, r)$ is rd-continuous and $V \oplus W \in \Re^{+}$. Therefore, inequality (14) with the use of Lemma 2.3 and (11) implies the estimate

$$
A(\zeta, r) \leq A\left(\zeta_{0}, r\right) e_{V \oplus W}\left(\zeta, \zeta_{0}\right)+\int_{\zeta_{0}}^{\zeta} e_{V \oplus W}(\tau, \sigma(o)) D(o, r) \Delta o .
$$

Substituting (9) and (15) on the right side of (11), we have

$$
A\left(\zeta_{0}, r\right) \leq \frac{\left.\int_{\zeta_{0}}^{S} m(o, r)\left[y(o, r)+\int_{\zeta_{0}}^{o} e_{V \oplus W}(o, \sigma(\tau)) D(\tau, r) \Delta \tau\right)\right] \Delta o}{1-\int_{\zeta_{0}}^{S} m(o, r) e_{V \oplus W}\left(o, \zeta_{0}\right) \Delta o},
$$

and, from (9), (15), and (16), we get the required bound in (3).

A useful corollary of Theorem 3.1 with $m(\zeta, r)=0$ can be obtained as follows:

Corollary 3.2 Under the same assumptions on $x, y, l, t, u, z, c, \xi, \psi, \Pi, \Theta, V, W, \eta$, and $H$ in Theorem 3.1, the inequality

$$
\begin{aligned}
& x(\zeta, r) \\
& \quad \leq y(\zeta, r)+\int_{\zeta_{0}}^{\zeta} \int_{r_{0}}^{r} l(o, \varrho) x(o, \varrho) \Delta \varrho \Delta o+t(\zeta, r) \int_{\zeta_{0}}^{\zeta} \int_{r_{0}}^{r} \Pi(\zeta, r, o, \varrho) \\
& \quad \times\left[u(o, \varrho) x(o, \varrho)+z(o, \varrho) x^{\xi}(\sigma(o), \varrho)-c(o, \varrho) x^{\psi}(\sigma(o), \varrho)\right] \Delta \varrho \Delta o, \quad(\zeta, r) \in \Upsilon,
\end{aligned}
$$

implies

$$
x(\zeta, r) \leq y(\zeta, r)+\int_{\zeta_{0}}^{\zeta} e_{V \oplus W}(\tau, \sigma(o)) D(o, r) \Delta o,
$$


If $\Pi(\zeta, r, o, \varrho)=1$ in Theorem 3.1, we can produce the following corollary:

Corollary 3.3 Suppose that $x, y, l, t, u, m, z, c, \xi, \psi$ and $H$ are same as in Theorem 3.1. If

$$
\begin{aligned}
x(\zeta, r) \leq & y(\zeta, r)+\int_{\zeta_{0}}^{\zeta} \int_{r_{0}}^{r} l(o, \varrho) x(o, \varrho) \Delta \varrho \Delta o \\
& +t(\zeta, r) \int_{\zeta_{0}}^{\zeta} \int_{r_{0}}^{r}\left[u(o, \varrho) x(o, \varrho)+z(o, \varrho) x^{\xi}(\sigma(o), \varrho)-c(o, \varrho) x^{\psi}(\sigma(o), \varrho)\right] \Delta \varrho \Delta o \\
& +\int_{\zeta_{0}}^{S} m(o, r) x(o, r) \Delta o, \quad(\zeta, r) \in \Upsilon,
\end{aligned}
$$

then

$$
\begin{aligned}
x(\zeta, r) \leq & y(\zeta, r)+\frac{\int_{\zeta_{0}}^{S} m(a, r)\left[y(o, r)+\int_{\zeta_{0}}^{o} e_{V_{1} \oplus W_{1}}(o, \sigma(\tau)) D_{1}(\tau, r) \Delta \tau\right] \Delta o}{1-\int_{\zeta 0}^{S} m(o, r) e_{V_{1} \oplus W_{1}}\left(o, \zeta_{0}\right) \Delta o} e_{V_{1} \oplus W_{1}}\left(\zeta, \zeta_{0}\right) \\
& +\int_{\zeta_{0}}^{S} m(o, r) e_{V_{1} \oplus W_{1}}(\tau, \sigma(o)) \Delta o
\end{aligned}
$$

where $V_{1}(\zeta, r)=\frac{\Theta_{1}(\zeta, r)}{1-\mu(\zeta, r) \Theta_{1}(\zeta, r)}, D_{1}(\zeta, r)=\left(1+\mu(\zeta, r) V_{1}(\zeta, r)\right) \eta_{1}(\zeta, r)$

$$
\begin{aligned}
\Theta_{1}(\zeta, r)= & t^{\Delta_{1}}(\zeta, r) \int_{\zeta_{0}}^{\sigma(\zeta)} \int_{r_{0}}^{r}(u(o, \varrho)+H(o, \varrho)) \Delta \varrho \Delta o+t(\zeta, r) \int_{r_{0}}^{r} H(\zeta, \varrho) \Delta \varrho, \\
W_{1}(\zeta, r)= & \int_{r_{0}}^{r} l(\zeta, \varrho) \Delta \varrho+t(\zeta, r) \int_{r_{0}}^{r} u(\zeta, \varrho) \Delta \varrho, \\
\eta_{1}(\zeta, r)= & \int_{r_{0}}^{r} l(\zeta, \varrho) y(\zeta, \varrho) \Delta v \varrho \\
& +t^{\Delta_{1}}(\zeta, r) \int_{\zeta 0}^{\sigma(\zeta)} \int_{r_{0}}^{r}(u(o, \varrho) y(o, \varrho)+H(o, \varrho) y(\sigma(\varrho), o)) \Delta \varrho \Delta o \\
& +t(\zeta, r) \int_{r_{0}}^{r}(u(\zeta, \varrho) y(\zeta, \varrho)+H(\zeta, \varrho) y(\sigma(\zeta), \varrho)) \Delta \varrho .
\end{aligned}
$$

Remark 3.4 Theorem 3.1 yields Lemma 3.1 of [33] by taking $x(\zeta, r)=u(t), y(\zeta, r)=m(t)$, $t(\zeta, r)=l(t), l(\zeta, r)=z(\zeta, r)=c(\zeta, r)=m(\zeta, r)=0, \Pi(\zeta, r, o, \varrho)=1, u(\zeta, r)=n(t)$ for fixed $r$ and $m(t)$ being a nondecreasing function.

Remark 3.5 As a distinctive case with $r$ fixed, Theorem 3.1 reduces to Theorem 5 of [34] if we put $x(\zeta, r)=x^{\beta}(t), \beta \geq 1$ being a constant, $y(\zeta, r)=a(t), l(\zeta, r)=b(t), t(\zeta, r)=1, z(\zeta, r)=$ $1, u(\zeta, r)=m(\zeta, r)=c(\zeta, r)=0, \Pi(\zeta, r, o, \varrho)=1, x(\sigma(\zeta), r)=L(t, x(\tau(s)))$ and $\xi=1$.

Theorem 3.6 Assume that $x, y, l, t, u, m, z, c, \xi, \psi, \Pi, \Pi^{\Delta_{1}}, \Pi^{\Delta_{2} \Delta_{1}}$ and $H$ are as in Theorem 3.1. Also let

$$
\begin{aligned}
x(\zeta, r) \leq & y(\zeta, r)+\int_{\zeta_{0}}^{\zeta} \int_{r_{0}}^{r} l(o, \varrho) x(\sigma(o), \varrho) \Delta \varrho \Delta o \\
& +t(\zeta, r) \int_{\zeta_{0}}^{\zeta} \int_{r_{0}}^{r} \Pi(\zeta, r, o, \varrho)\left[u(o, \varrho) x(o, \varrho)+z(o, \varrho) x^{\xi}(\sigma(o), \varrho)\right.
\end{aligned}
$$




$$
\begin{aligned}
& \left.-c(o, \varrho) x^{\psi}(\sigma(o), \varrho)\right] \Delta \varrho \Delta o \\
& +t(S, R) \int_{\zeta_{0}}^{S} \int_{r_{0}}^{R} \Pi(\zeta, r, o, \varrho)\left[u(o, \varrho) x(o, \varrho)+z(o, \varrho) x^{\xi}(\sigma(o), \varrho)\right. \\
& \left.-c(o, \varrho) x^{\psi}(\sigma(o), \varrho)\right] \Delta \varrho \Delta o,
\end{aligned}
$$

for $(p, r) \in \Upsilon$. If

$$
\begin{aligned}
\Omega & =\int_{\zeta_{0}}^{\zeta} \int_{r_{0}}^{r} \Pi(\zeta, r, o, \varrho)\left[u(o, \varrho) e_{V_{2} \oplus W_{2}}\left(o, \zeta_{0}\right)+H(o, \varrho) e_{V_{2} \oplus W_{2}}\left(\sigma(o), \zeta_{0}\right)\right] \Delta \varrho \Delta o \\
& <\frac{1}{t(\zeta, r)},
\end{aligned}
$$

then

$$
x(\zeta, r) \leq \frac{y(S, R)}{1-\Omega t(S, R)} e_{V_{2} \oplus W_{2}}\left(\zeta, \zeta_{0}\right),
$$

so that

$$
\begin{aligned}
\Theta_{2}(\zeta, r)= & \int_{r_{0}}^{r} l(\zeta, \varrho) \Delta \zeta+t^{\Delta_{1}}(\zeta, r) \int_{\zeta_{0}}^{\sigma(\zeta)} \int_{r_{0}}^{r} \Pi(\sigma(\zeta), r, o, \varrho)(u(o, \varrho)+H(o, \varrho)) \Delta \varrho \Delta o \\
& +t(\zeta, r) \int_{\zeta_{0}}^{\zeta} \int_{r_{0}}^{r} \Pi^{\Delta_{2} \Delta_{1}}(\zeta, r, o, \varrho) H(o, \varrho) \Delta \varrho \Delta o \\
& +\int_{r_{0}}^{r} \Pi^{\Delta_{1}}(\sigma(\zeta), r, \zeta, v) H(\zeta, \varrho) \Delta \varrho \\
W_{2}(\zeta, r)= & t(\zeta, r) \int_{\zeta_{0}}^{\zeta} \int_{r_{0}}^{r} \Pi^{\Delta_{2} \Delta_{1}}(\zeta, r, o, \varrho) u(o, \varrho) \Delta \varrho \Delta o \\
& +\int_{r_{0}}^{r} \Pi^{\Delta_{1}}(\sigma(\zeta), r, \zeta, \varrho) u(\zeta, \varrho) \Delta \varrho \\
V_{2}(\zeta, r)= & \frac{\Theta \Theta_{2}(\zeta, r)}{1-\mu(\zeta, r) \Theta_{2}(\zeta, r)}
\end{aligned}
$$

Proof Inequality (17), by applying Lemma 2.2, gives

$$
\begin{aligned}
x(\zeta, r)= & y(\zeta, r)+\int_{\zeta_{0}}^{\zeta} \int_{r_{0}}^{r} l(o, \varrho) x(\sigma(o), \varrho) \Delta \varrho \Delta o \\
& +t(\zeta, r) \int_{\zeta_{0}}^{\zeta} \int_{r_{0}}^{r} \Pi(\zeta, r, o, \varrho)\left[u(o, \varrho) x(o, \varrho)+z(o, \varrho) x^{\xi}(\sigma(o), \varrho)\right. \\
& \left.-c(o, \varrho) x^{\psi}(\sigma(o), \varrho)\right] \Delta \varrho \Delta o \\
& +t(S, R) \int_{\zeta_{0}}^{S} \int_{r_{0}}^{R} \Pi(\zeta, r, o, \varrho)\left[u(o, \varrho) x(o, \varrho)+z(o, \varrho) x^{\xi}(\sigma(o), \varrho)\right. \\
& \left.-c(o, \varrho) x^{\psi}(\sigma(o), \varrho)\right] \Delta \varrho \Delta o \\
\leq & y(\zeta, r)+\int_{\zeta_{0}}^{\zeta} \int_{r_{0}}^{r} l(o, \varrho) x(\sigma(o), \varrho) \Delta \varrho \Delta o
\end{aligned}
$$




$$
\begin{aligned}
& +t(\zeta, r) \int_{\zeta_{0}}^{\zeta} \int_{r_{0}}^{r} \Pi(\iota, r, o, \varrho)[u(o, \varrho) x(o, \varrho)+H(o, \varrho) x(\sigma(o), \varrho)] \Delta \varrho \Delta o \\
& +t(S, R) \int_{\zeta_{0}}^{S} \int_{r_{0}}^{R} \Pi(\zeta, r, o, \varrho)[u(o, \varrho) x(o, \varrho)+H(o, \varrho) x(\sigma(o), \varrho)] \Delta \varrho \Delta o .
\end{aligned}
$$

Denote $A_{1}(\zeta, r)$ a nondecreasing function as

$$
\begin{aligned}
A_{1}(\zeta, r)= & y(S, R)+\int_{\zeta_{0}}^{\zeta} \int_{r_{0}}^{r} l(o, \varrho) x(\sigma(o), \varrho) \Delta \varrho \Delta o \\
& +t(\zeta, r) \int_{\zeta_{0}}^{\zeta} \int_{r_{0}}^{r} \Pi(\zeta, r, o, \varrho)[u(o, \varrho) x(o, \varrho)+H(o, \varrho) x(\sigma(o), \varrho)] \Delta \varrho \Delta o \\
& +t(S, R) \int_{\zeta_{0}}^{S} \int_{r_{0}}^{R} \Pi(S, R, o, \varrho)[u(o, \varrho) x(o, \varrho)+H(o, \varrho) x(\sigma(o), \varrho)] \Delta \varrho \Delta o,
\end{aligned}
$$

then, from (23) and (24), we get

$$
x(\zeta, r) \leq A_{1}(\zeta, r)
$$

and

$$
\begin{aligned}
A_{1}\left(\zeta_{0}, r\right)= & y(S, R)+t(S, R) \int_{\zeta_{0}}^{S} \int_{r_{0}}^{R} \Pi(S, R, o, \varrho)[u(o, \varrho) x(o, \varrho) \\
& +H(o, \varrho)) x(\sigma(o), \varrho)] \Delta \varrho \Delta o .
\end{aligned}
$$

Using Lemma 2.1 and delta differentiation (24) with respect to $j$, we have

$$
\begin{aligned}
& A_{1}^{\Delta_{1}}(\zeta, r) \\
& \quad \leq \int_{r_{0}}^{r} l(\zeta, \varrho) x(\sigma(\zeta), \varrho) \Delta \varrho \\
& \quad+t^{\Delta_{1}}(\zeta, r) \int_{\zeta_{0}}^{\sigma(\zeta)} \int_{r_{0}}^{r} \Pi(\sigma(\zeta), r, o, \varrho)[u(o, \varrho) x(o, \varrho)+H(o, \varrho) x(\sigma(o), \varrho)] \Delta \varrho \Delta o \\
& \quad+t(\zeta, r) \int_{\zeta 0}^{\zeta} \int_{r_{0}}^{r} \Pi^{\Delta_{2} \Delta_{1}}(\zeta, r, o, \varrho)[u(o, \varrho) x(o, \varrho)+H(o, \varrho) x(\sigma(o), \varrho)] \Delta \varrho \Delta o \\
& \quad+t(\zeta, r) \int_{r_{0}}^{r} \Pi^{\Delta_{1}}(\sigma(\zeta), r, \zeta, \varrho)[u(p, \varrho) x(p, \varrho)+H(p, \varrho) x(\sigma(\zeta), \varrho)] \Delta \varrho .
\end{aligned}
$$

The above inequality with the help of (25) takes the form of

$$
\begin{aligned}
& A_{1}^{\Delta_{1}}(\zeta, r) \\
& \quad \leq \int_{r_{0}}^{r} l(\zeta, \varrho) A_{1}(\sigma(\zeta), \varrho) \Delta \varrho \\
& \quad+t^{\Delta_{1}}(\zeta, r) \int_{\zeta_{0}}^{\sigma(\zeta)} \int_{r_{0}}^{r} \Pi(\sigma(\zeta), r, o, \varrho)\left[u(o, \varrho) A_{1}(o, \varrho)+H(o, \varrho) A_{1}(\sigma(o), \varrho)\right] \Delta \varrho \Delta o \\
& \quad+t(\zeta, r) \int_{\zeta 0}^{\zeta} \int_{r_{0}}^{r} \Pi^{\Delta_{2} \Delta_{1}}(\zeta, r, o, \varrho)\left[u(o, \varrho) A_{1}(o, \varrho)+H(o, \varrho) A_{1}(\sigma(o), \varrho)\right] \Delta \varrho \Delta o
\end{aligned}
$$




$$
\begin{aligned}
& +t(\zeta, r) \int_{r_{0}}^{r} \Pi^{\Delta_{1}}(\sigma(\zeta), r, \zeta, \varrho)\left[u(\zeta, \varrho) A_{1}(\zeta, \varrho)+H(\zeta, \varrho) A_{1}(\sigma(\zeta), \varrho)\right] \Delta \varrho \\
\leq & \Theta_{2}(\zeta, r) A_{1}(\sigma(\zeta), r)+W_{2}(\zeta, r) A_{1}(\zeta, r)
\end{aligned}
$$

with $\Theta_{2}(\zeta, r), W_{2}(\zeta, r)$ as in (20) and (21), respectively. It can be observed from (22) that

$$
\Theta_{2}(\zeta, r)=\frac{V_{2}(\zeta, r)}{1+\mu(\zeta, r) V_{2}(\zeta, r)}
$$

Substituting (28) in (27), we acquire

$$
\begin{aligned}
A_{1}^{\Delta_{1}}(\zeta, r) & \leq \Theta_{2}(\zeta, r)\left(A_{1}(\zeta, r)+\mu(\zeta, r) A_{1}^{\Delta_{1}}(\zeta, r)\right)+W_{2}(\zeta, r) A_{1}(\zeta, r) \\
& \leq \frac{V_{2}(\zeta, r)}{1+\mu(\zeta, r) V_{2}(\zeta, r)}\left(A_{1}(\zeta, r)+\mu(\zeta, r) A_{1}^{\Delta_{1}}(\zeta, r)\right)+W_{2}(\zeta, r) A_{1}(\zeta, r)
\end{aligned}
$$

which implies

$$
\begin{aligned}
A_{1}^{\Delta_{1}}(\zeta, r) \leq & \left(V_{2}(\zeta, r)+W_{2}(\zeta, r)+\mu(\zeta, r) V_{2}(\zeta, r) W_{2}(\zeta, r)\right) A_{1}(\zeta, r) \\
& +\left(1+\mu(\zeta, r) V_{2}(\zeta, r)\right) \\
\leq & \left(V_{2} \oplus W_{2}\right)(\zeta, r) A_{1}(\zeta, r)
\end{aligned}
$$

Since $A_{1}(\zeta, r)$ is rd-continuous and $V_{2} \oplus W_{2} \in \Re^{+}$, inequality (29), with Lemma 2.4, (25) and (26), produces

$$
\begin{aligned}
A_{1}(\zeta, r) \leq & A_{1}\left(\zeta_{0}, r\right) e_{V_{2} \oplus W_{2}}\left(\zeta, \zeta_{0}\right) \\
\leq & \left\{y(S, R)+t(S, R) \int_{\zeta_{0}}^{S} \int_{r_{0}}^{R} \Pi(S, R, o, \varrho)\left[u(o, \varrho) A_{1}(o, \varrho)\right.\right. \\
& \left.\left.+H(o, \varrho)) A_{1}(\sigma(o), \varrho)\right] \Delta \varrho \Delta o\right\} e_{V_{2} \oplus W_{2}}\left(\zeta, \zeta_{0}\right) \\
= & B(S, R) e_{V_{2} \oplus W_{2}}\left(\zeta, \zeta_{0}\right),
\end{aligned}
$$

where

$$
\begin{aligned}
B(S, R)= & y(S, R) \\
& \left.+t(S, R) \int_{\zeta_{0}}^{S} \int_{r_{0}}^{R} \Pi(S, R, o, \varrho)\left[u(o, \varrho) A_{1}(o, \varrho)+H(o, \varrho)\right) A_{1}(\sigma(o), \varrho)\right] \Delta \varrho \Delta o \\
\leq & y(S, R)+t(S, R) \int_{\zeta_{0}}^{S} \int_{r_{0}}^{R} \Pi(S, R, o, \varrho) \\
& \left.\times\left[u(o, \varrho) B(S, R) e_{V_{2} \oplus W_{2}}\left(o, \zeta_{0}\right)+H(o, \varrho)\right) B(\sigma(S), R) e_{V_{2} \oplus W_{2}}\left(\sigma(o), \zeta_{0}\right)\right] \Delta \varrho \Delta o \\
\leq & \frac{y(S, R)}{1-\Omega t(S, R)} .
\end{aligned}
$$

Here $\Omega$ is defined as in (18). The conclusion in (19) can be achieved from (25), (30), and (31). Details are omitted. 
Remark 3.7 If $x(\zeta, r)=u^{\beta}(x, y), y(\zeta, r)=c, c>0, t(\zeta, r)=0, l(\zeta, r)=f(x, y), x(\sigma(\zeta), r)=$ $\omega\left(u\left(\tau_{1}(t), \tau_{2}(t)\right)\right)$, then Theorem 3.6 can be transformed into the inequality of Theorem 2.14 stated by Zheng et al. [35].

Remark 3.8 If $x(\zeta, r)=x(\sigma(\zeta), r)=u(t, s), y(p, r)=a(t, s), l(\zeta, r)=b(t, s)$ and $t(\zeta, r)=0$, then we can conveniently reach Theorem 1 of [36] from Theorem 3.6.

Theorem 3.9 Let $x, y, l, u, z, c, H, \xi$ and $\psi$ be as in Theorem 3.1. In addition, suppose $m \in$ $C_{r d}\left(\Upsilon \times \Upsilon, \mathbb{R}_{+}\right)$is nondecreasing in $j$ and

$$
\begin{aligned}
x(\zeta, r) \leq & y(\zeta, r)+\int_{\zeta_{0}}^{\zeta} \int_{r_{0}}^{r} l(\zeta, r, o, \varrho)[x(o, \varrho) \\
& \left.+\int_{\zeta_{0}}^{o} \int_{r_{0}}^{\varrho}\left(u(n, k) x(\sigma(n), k)+z(n, k) x^{\xi}(n, k)-c(n, k) x^{\psi}(n, k)\right) \Delta k \Delta n\right] \Delta \varrho \Delta o \\
& +\int_{\zeta_{0}}^{S} m(\zeta, o, r) x(o, r) \Delta o, \quad(\zeta, r) \in \Upsilon
\end{aligned}
$$

with

$$
\int_{\zeta_{0}}^{S} m(S, o, r) e_{V_{3} \oplus W_{3}}\left(o, \zeta_{0}\right) \Delta o<1
$$

Then

$$
x(\zeta, r) \leq \frac{y(S, r)}{1-\int_{\zeta_{0}}^{S} m(S, o, r) e_{V_{3} \oplus W_{3}}\left(o, \zeta_{0}\right) \Delta o} e_{V_{3} \oplus W_{3}}\left(\zeta, \zeta_{0}\right),
$$

where

$$
\begin{aligned}
\Theta_{3}(\zeta, r)= & \int_{r_{0}}^{r} u(\zeta, \varrho) \Delta \varrho \\
W_{3}(\zeta, r)= & \int_{r_{0}}^{r} l(\sigma(\zeta), r, \zeta, \varrho) \Delta \varrho+\int_{\zeta_{0}}^{\zeta} \int_{r_{0}}^{r} l^{\Delta_{2} \Delta_{1}}(\zeta, r, o, \varrho) \Delta \varrho \Delta o \\
& +\int_{r_{0}}^{r} H(\zeta, \varrho) \Delta \varrho \\
V_{3}(\zeta, r)= & \frac{\Theta_{3}(\zeta, r)}{1-\mu(\zeta, r) \Theta_{3}(\zeta, r)} .
\end{aligned}
$$

Proof From (32) and Lemma 2.2, we have

$$
\begin{aligned}
x(\zeta, r) \leq & y(\zeta, r)+\int_{\zeta_{0}}^{\zeta} \int_{r_{0}}^{r} l(\zeta, r, o, \varrho)[x(o, \varrho) \\
& \left.+\int_{\zeta_{0}}^{o} \int_{r_{0}}^{\varrho}(u(n, k) x(\sigma(n), k)+H(n, k) x(n, k)) \Delta k \Delta n\right] \Delta \varrho \Delta o \\
& +\int_{\zeta_{0}}^{S} m(\zeta, o, r) x(o, r) \Delta o .
\end{aligned}
$$


Clearly, $x(\zeta, r)$ is nonnegative. Also $\int_{\zeta_{0}}^{S} m(\zeta, o, r) x(o, r) \Delta o$ is a nondecreasing function because $m(\zeta, o, \varrho)$ is a nondecreasing function for $(\zeta, r) \in \Upsilon$, By delta-differentiating (38), we get

$$
\begin{aligned}
x^{\Delta_{1}}(\zeta, r)= & \int_{r_{0}}^{r} l(\sigma(\zeta), r, \zeta, \varrho)\left[x(\zeta, \varrho)+\int_{\zeta_{0}}^{\zeta} \int_{r_{0}}^{\varrho}(u(o, k) x(\sigma(o), k)\right. \\
& +H(o, k) x(o, k) \Delta k \Delta o)] \Delta \varrho \\
& +\int_{\zeta_{0}}^{\zeta} \int_{r_{0}}^{r} l^{\Delta_{2} \Delta_{1}}(\zeta, r, o, \varrho)[x(o, \varrho) \\
& \left.+\int_{\zeta_{0}}^{o} \int_{r_{0}}^{v}(u(n, k) x(\sigma(n), k)+H(n, k) x(n, k)) \Delta k \Delta n\right] \Delta \varrho \Delta o \\
\leq & {\left[\int_{r_{0}}^{r} l(\sigma(\zeta), r, \zeta, \varrho) \Delta v+\int_{\zeta_{0}}^{\zeta} \int_{r_{0}}^{r} l^{\Delta_{2} \Delta_{1}}(\zeta, r, o, \varrho) \Delta \varrho \Delta o\right] A_{2}(\zeta, r), }
\end{aligned}
$$

so that

$$
A_{2}(\zeta, r)=x(\zeta, r)+\int_{\zeta_{0}}^{\zeta} \int_{r_{0}}^{r}(u(o, \varrho) x(\sigma(o), \varrho)+H(o, \varrho) x(o, \varrho)) \Delta \varrho \Delta o
$$

and

$$
x(\zeta, r) \leq A_{2}(\zeta, r)
$$

From inequality (39), again by delta differentiating with respect to $j$ and using (40) and (41), we get

$$
A_{2}^{\Delta_{1}}(\zeta, r) \leq W_{3}(\zeta, r) A_{2}(\zeta, r)+\Theta_{3}(\zeta, r) A_{2}(\sigma(\zeta), r)
$$

Further, the above inequality, by utilizing Lemma 2.4, (37), and the fact that $A_{2}\left(\zeta_{0}, r\right)=$ $y(\zeta, r)+\int_{\zeta_{0}}^{P} m(\zeta, o, r) x(o, r) \Delta o$, gives the bound

$$
A_{2}(\zeta, r) \leq\left(y(\zeta, r)+\int_{\zeta_{0}}^{S} m(\zeta, o, r) x(o, r) \Delta o\right) e_{V_{3} \oplus W_{3}}\left(\zeta, \zeta_{0}\right),
$$

where $\Theta_{3}, W_{3}, V_{3}$ are defined as in (35), (36), and (37), respectively. The monotonicity of $y$ and $m$ in the last inequality yields

$$
\begin{aligned}
A_{2}(\zeta, r) & \leq\left(y(S, r)+\int_{\zeta_{0}}^{P} m(S, o, r) x(o, r) \Delta o\right) e_{V_{3} \oplus W_{3}}\left(\zeta, \zeta_{0}\right) \\
& =B_{1}(S, r) e_{V_{3} \oplus W_{3}}\left(\zeta, \zeta_{0}\right)
\end{aligned}
$$

where

$$
B_{1}(S, r)=y(S, r)+\int_{\zeta_{0}}^{S} m(S, o, r) x(o, r) \Delta o .
$$


From the definition of $B_{1}(\zeta, r),(41)$ and (42), we obtain

$$
B_{1}(S, r) \leq \frac{y(S, r)}{1-\int_{\zeta_{0}}^{S} m(S, o, r) e_{V_{3} \oplus W_{3}}\left(o, \zeta_{0}\right) \Delta o},
$$

so the required bound in (34) can be acquired by combining (41), (42), and (43).

Remark 3.10 If we fix $r$ and $x(\zeta, r)=u(t), y(\zeta, r)=c, c \geq 0, l(\zeta, r, o, \varrho)=f(t), u(\zeta, r)=$ $c(\zeta, r)=m(\zeta, r)=0, z(\zeta, r)=\omega(t, s)$ in Theorem 3.9, where $\omega(t, s) \geq 0$, then we get the inequality obtained by Li et al. in [37]; see their Theorem 3.1.

\section{Applications}

In this section we focus on some of the applications of Theorem 3.1 to research certain properties of differential equation solutions. Let us discuss the following dynamic integral equation on time scales:

$$
\begin{aligned}
x(\zeta, r)= & y(\zeta, r)+\int_{\zeta_{0}}^{\zeta} \int_{r_{0}}^{r} Y(o, \varrho, x(o, \varrho)) \Delta \varrho \Delta o \\
& +t(\zeta, r) \int_{\zeta_{0}}^{\zeta} \int_{r_{0}}^{r} P(\zeta, r, o, \varrho, x(o, \varrho), x(\sigma(o), \varrho)) \Delta \varrho \Delta o \\
& +\int_{\zeta_{0}}^{\zeta} T(o, r, x(o, r)) \Delta o,
\end{aligned}
$$

where $P \in C_{r d}\left(\Upsilon^{2} \times \mathbb{R}^{2}, \mathbb{R}\right)$ and $Y, T \in C_{r d}(\Upsilon \times \mathbb{R}, \mathbb{R})$.

The ensuing example addresses the global existence on the solutions of (44).

Example 4.1 Suppose that

$$
\begin{aligned}
& |Y(\zeta, r, d)| \leq l(\zeta, r)|d|, \\
& |P(\zeta, r, o, \varrho, d, h)| \leq \Pi(\zeta, r, o, \varrho)\left[u(o, \varrho)|d|+z(o, \varrho)|h|^{\xi}-c(o, \varrho)|h|^{\psi}\right], \\
& |T(\zeta, r, d)| \leq m(\zeta, r)|d|,
\end{aligned}
$$

for $(\zeta, r) \in \Upsilon, d, \varrho \in \mathbb{R}$, then $x(\zeta, r)$ is a solution of (44) with

$$
\mu(\zeta, r) \Theta(\zeta, r)<1, \quad \int_{\zeta_{0}}^{S} m(o, r) e_{V \oplus W}\left(o, \zeta_{0}\right) \Delta o<1
$$

and satisfies

$$
\begin{aligned}
|x(\zeta, r)| \leq & |y(\zeta, r)|+\frac{\int_{\zeta_{0}}^{S} m(o, r)\left[|y(o, r)|+\int_{\zeta_{0}}^{o} e_{V \oplus W}(o, \sigma(\tau)) D(\tau, r) \Delta \tau\right] \Delta a}{1-\int_{\zeta_{0}}^{S} m(o, r) e_{V \oplus W}\left(o, \zeta_{0}\right) \Delta o} e_{V \oplus W}\left(\zeta, \zeta_{0}\right) \\
& +\int_{\zeta_{0}}^{S} m(o, r) e_{V \oplus W}(\tau, \sigma(o)) \Delta o
\end{aligned}
$$

where $x, l, t, y, u, z, c, m, \Pi, \xi, \psi, \Theta, W, V$ and $D$ are as in Theorem 3.1. 
Proof Equation (44) by utilizing (45)-(47) can be restated as

$$
\begin{aligned}
|x(\zeta, r)| \leq & \mid y(\zeta, r)+\int_{\zeta_{0}}^{S} \int_{\left.r_{0}\right)}^{r} Y(o, \varrho, x(o, \varrho)) \Delta \varrho \Delta o \\
& +t(\zeta, r) \int_{\zeta_{0}}^{p} \int_{r_{0}}^{r} P(\zeta, r, o, \varrho, x(o, \varrho), x(\sigma(o), \varrho)) \Delta v \Delta o \\
& +\int_{\zeta 0}^{\zeta} T(o, r, x(o, r)) \Delta o \mid \\
\leq & |y(\zeta, r)|+\int_{\zeta_{0}}^{\zeta} \int_{r_{0}}^{r}|Y(o, \varrho, x(o, \varrho))| \Delta \varrho \Delta o \\
& +t(\zeta, r) \int_{\zeta_{0}}^{\zeta} \int_{r_{0}}^{r}|P(\zeta, r, o, \varrho, x(o, \varrho), x(\sigma(o), \varrho))| \Delta \varrho \Delta o \\
& +\int_{\zeta 0}^{\zeta}|T(o, r, x(o, r))| \Delta o \\
\leq & |y(\zeta, r)|+\int_{\zeta_{0}}^{\zeta} \int_{r_{0}}^{r} l(o, \varrho)|x(o, \varrho)| \Delta \varrho \Delta o \\
& +t(\zeta, r) \int_{\zeta_{0}}^{\zeta} \int_{r_{0}}^{r} \Pi(\zeta, r, o, \varrho)\left[u(o, \varrho)|x(o, \varrho)|+z(o, \varrho)|x(\sigma(o), \varrho)|^{\xi}\right. \\
& +c(o, \varrho)|x(\sigma(o), \varrho)| \psi] \Delta \varrho \Delta o \\
& +\int_{\zeta_{0}}^{S} m(o, r)|x(o, r)| \Delta o . \\
& \\
& \\
&
\end{aligned}
$$

We attain (48) by applying the same procedure as in the proof of Theorem 3.1 to (49).

Example 4.2 Let us consider the following hypotheses:

$$
\begin{aligned}
& \left|Y\left(\zeta, r, d_{1}\right)-Y\left(\zeta, r, d_{2}\right)\right| \leq l(\zeta, r)\left|d_{1}-d_{2}\right|, \\
& \left|P\left(\zeta, r, o, \varrho, d_{1}, h_{1}\right)-P\left(\zeta, r, o, \varrho, d_{2}, h_{2}\right)\right| \leq \Pi(\zeta, r, o, \varrho)\left[u(o, \varrho)\left|d_{1}-d_{2}\right|\right. \\
& \left.+z(o, \varrho)\left|h_{1}-h_{2}\right|^{\xi}-c(o, \varrho)\left|h_{1}-h_{2}\right|^{\psi}\right], \\
& \left|T\left(\zeta, r, d_{1}\right)-T\left(\zeta, r, d_{2}\right)\right| \leq m(\zeta, r)\left|d_{1}-d_{2}\right|,
\end{aligned}
$$

then the dynamic integral equation (44) has a unique solution.

Proof If $x_{1}(\zeta, r)$ and $x_{2}(\zeta, r)$ are solutions of (44), then

$$
\begin{aligned}
x_{1}(\zeta, r)-x_{2}(\zeta, r)= & \int_{\zeta_{0}}^{\zeta} \int_{r_{0}}^{r}\left[Y\left(o, \varrho, x_{1}(o, \varrho)\right)-Y\left(o, \varrho, x_{2}(o, \varrho)\right)\right] \Delta v \Delta o \\
& +t(\zeta, r) \int_{\zeta_{0}}^{\zeta} \int_{r_{0}}^{r}\left[P\left(\zeta, r, o, \varrho, x_{1}(o, \varrho), x_{1}(\sigma(o), \varrho)\right)\right. \\
& \left.-P\left(\zeta, r, o, \varrho, x_{2}(o, \varrho), x_{2}(\sigma(o), \varrho)\right)\right] \Delta \varrho \Delta o \\
& +\int_{\zeta_{0}}^{\zeta}\left[T\left(o, r, x_{1}(o, r)\right)-T\left(o, r, x_{1}(o, r)\right)\right] \Delta o .
\end{aligned}
$$


From (50)-(52) used to the previous equation, we get

$$
\begin{aligned}
& \left|x_{1}(\zeta, r)-x_{2}(\zeta, r)\right| \\
& \leq \int_{\zeta_{0}}^{\zeta} \int_{r_{0}}^{r} l(o, \varrho)\left|x_{1}(o, \varrho)-x_{2}(o, \varrho)\right| \Delta \varrho \Delta o+t(\zeta, r) \int_{\zeta_{0}}^{\zeta} \int_{r_{0}}^{r} \Pi(\zeta, r, o, \varrho) \\
& \quad \times\left[u(o, \varrho)\left|x_{1}(o, \varrho)-x_{2}(o, \varrho)\right|+z(o, \varrho)\left|x_{1}(\sigma(o), \varrho)-x_{2}(\sigma(o), \varrho)\right|^{\xi}\right. \\
& \left.\quad-c(o, \varrho)\left|x_{1}(\sigma(o), \varrho)-x_{2}(\sigma(o), \varrho)\right|^{\psi}\right] \Delta \varrho \Delta o \\
& \quad+\int_{\zeta_{0}}^{S} m(o, r)\left|x_{1}(o, r)-x_{2}(o, r)\right| \Delta o,
\end{aligned}
$$

so applying a similar method as that in Theorem 3.1 with suitable changes to the function $\left|x_{1}(p, r)-x_{2}(p, r)\right|$ in (53), we have

$$
\left|x_{1}(\zeta, r)-x_{2}(\zeta, r)\right| \leq 0, \quad(\zeta, r) \in \Upsilon
$$

Hence $x_{1}(\zeta, r)=x_{2}(\zeta, r)$. Thus the dynamic equation (44) has one positive solution.

\section{Conclusion}

Like other recognized and proven inequalities in the literature, Theorems 3.1, 3.6, and 3.9 examined such dynamic integral inequalities with suggested remarks for two independent variables on time scales. In order to overcome the conceptual classifications of differential equations, one may also apply the introduced corollaries. Our results may be extended by using the basic methodology to solve the difficulty of achieving estimates on the specific bounds of unknown functions and therefore to expand and unify continuous inequalities. This investigation offers the foundation for further advancement of the idea of integral inequality for time scale calculus implementations, providing scope for maximizing efforts to reach a desirable outcome.

Acknowledgements

The authors would like to thank the editor and the referees for their careful reading and for their helpful comments and suggestions that led to considerable improvements of the paper.

\section{Funding}

This research was funded by the Deanship of Scientific Research at Princess Nourah bint Abdulrahman University through the Fast-track Research Funding Program.

\section{Abbreviations}

Not applicable.

Availability of data and materials

Not applicable.

Competing interests

The authors declare that there are no financial and nonfinancial competing interests.

Authors' contributions

The authors contributed equally in writing the final version of this article. All authors read and approved the final manuscript.

\section{Author details}

${ }^{1}$ Department of Mathematics, College of Science, Princess Nourah bint Abdulrahman University, Riyadh, Saudi Arabia. ${ }^{2}$ Department of Mathematics, Çankaya University, Ankara, Turkey. ${ }^{3}$ Department of Mathematics and General Sciences, Prince Sultan University, P.O. Box 66833, Riyadh, 11586, Saudi Arabia. ${ }^{4}$ Shaheed Benazir Bhutto University, Sheringal, 18000 Dir Upper, Khyber Pakhtunkhwa, Pakistan. 


\section{Publisher's Note}

Springer Nature remains neutral with regard to jurisdictional claims in published maps and institutional affiliations.

\section{Received: 12 June 2020 Accepted: 3 August 2020 Published online: 12 August 2020}

\section{References}

1. El-Owaidy, H., Ragab, A.A., Abuleela, W., El-Deeb, A.A.: On some new nonlinear integral inequalities of Gronwall-Bellman type. Kyungpook Math. J. 54, 555-575 (2014)

2. Kim, Y.H.: Gronwall, Bellman and Pachpatte type integral inequalities with applications. Nonlinear Anal., Theory Methods Appl. 71(12), e2641-e2656 (2009)

3. Khan, Z.: Further nonlinear version of inequalities and their applications. Filomat 33(18), 6005-6014 (2019)

4. Li, L., Meng, F., Ju, P.: Some new integral inequalities and their applications in studying the stability of nonlinear integro differential equations with time delay. J. Math. Anal. Appl. 377, 853-862 (2011)

5. Abdeljawad, T., Jarad, F., Baleanu, D.: Variational optimal-control problems with delayed arguments on time scales. Adv. Differ. Equ. 2009, 1 (2009)

6. Khan, H., Abdeljawad, T., Tun, C., Alkhazzan, A., Khan, A.: Minkowski's inequality for the AB-fractional integral operator. J. Inequal. Appl. 2019, 96 (2019)

7. Abdeljawad, T., Alzabut, J.: The 9 fractional analogue for Gronwall-type inequality. J. Funct. Spaces Appl. 2013, 9 (2013)

8. Kh, F.M., El-Deeb, A.A., Abdeldaim, A., Khan, Z.: On some generalizations of dynamic Opial-type inequalities on time scales. Adv. Differ. Equ. 2019, 323 (2019)

9. Khan, H., Jarad, F., Abdeljawad, T., Khan, A.: A singular ABC-fractional differential equation with $p$-Laplacian operator. Chaos Solitons Fractals 2019(1), 129 (2019)

10. Rashid, S., Jarad, F., Chu, Y.M.: A note on reverse Minkowski inequality via generalized proportional fractional integral operator with respect to another function. Math. Probl. Eng. 2020, Article ID 7630260 (2020)

11. Rashid, S., Jarad, F., Kalsoom, H., Chu, Y.M.: On Pólya, Szegö and Čhebyšev type inequalities via generalized k-fractional integrals. Adv. Differ. Equ. 2020, Article ID 125 (2020)

12. Rashid, S., Jarad, F., Noor, M.A., Kalsoom, H., Chu, Y.M.: Inequalities by means of generalized proportional fractional integral operators with respect another function. Mathematics 7(12), 1225 (2019)

13. Rashid, S., Noor, M.A., Noor, K.I., Chu, Y.M.: Ostrowski type inequalities in the sense of generalized k-fractional integral operator for exponentially convex functions. AIMS Math. 5(3), 2629-2645 (2020)

14. Zhou, S.S., Rashid, S., Jarad, F., Kalsoom, H., Chu, Y.M.: New estimates considering the generalized proportional Hadamard fractional integral operators. Adv. Differ. Equ. 2020, Article ID 275 (2020)

15. Khan, A., Khan, H., Gomez-Aguilar, J.F., Abdeljawad, T.: Existence and Hyers-Ulam stability for a nonlinear singular fractional differential equations with Mittag-Leffler kernel. Chaos Solitons Fractals 2019, 127 (2019)

16. Abdeljawad, T., Ullah, K., Ahmad, J., Malaiki, N.M.: Iterative approximation of endpoints for multivalued mappings in Banach spaces. J. Funct. Spaces 2020, Article ID 2179059 (2020)

17. Jarad, F., Abdeljawad, T., Shah, K.: On the weighted fractional operators of a function with respect to another function. Fractals (2020)

18. Hilger, S.: Analysis on measure chains - a unified approach to continuous and discrete calculus. Results Math. 18, 18-56 (1990)

19. Khan, Z.: On some explicit bounds of integral inequalities related to time scales. Adv. Differ. Equ. 2019, 243 (2019)

20. Khan, Z: Study on the estimates of Gronwall-Ou-lang dynamic integral inequalities by means of diamond- $\alpha$ derivatives. Adv. Differ. Equ. 2020, 240 (2020)

21. Khan, Z:: Solvability for a class of integral inequalities with maxima on the theory of time scales and their applications. Bound. Value Probl. 2019, 146 (2019)

22. Pachpatte, D.B.: Explicit estimates on integral inequalities with time scales. J. Inequal. Pure Appl. Math. 7(4), 143 (2006)

23. Li, W.N.: Some Pachpatte type inequalities on time scales. Comput. Math. Appl. 57, 275-282 (2009)

24. Sun, Y.G., Hassan, T.: Some nonlinear dynamic integral inequalities on time scales. Appl. Math. Comput. 220(4), 221-225 (2013)

25. Gu, J., Meng, F.W.: Some new nonlinear Volterra-Fredholm type dynamic integral inequalities on time scales. Appl. Math. Comput. 245(1), 235-242 (2014)

26. Khan, Z: Hadamard type fractional differential equations for the system of integral inequalities on time scales. Integral Transforms Spec. Funct. 31(5), 412-423 (2020)

27. $\mathrm{Mi}, \mathrm{Y}:$ A generalized Gronwall-Bellman type delay integral inequality with two independent variables on time scales. J. Math. Inequal. 11(4), 1151-1160 (2017)

28. Khan, Z: Analysis on some powered integral inequalities with retarded argument and application. J. Taibah Univ. Sci. 14(1), 488-495 (2020)

29. El-Deeb, A.A., Khan, Z: Certain new dynamic nonlinear inequalities in two independent variables and applications. Bound. Value Probl. 2020(1), 31 (2020)

30. Bohner, M.: Partial differentiation on time scales. In: Multivariable Dynamic Calculus on Time Scales, pp. 303-447 (2016)

31. Liu, H.D., Li, C.Y., Shen, F.C.: A class of new nonlinear dynamic integral inequalities containing integration on infinite interval on time scales. Adv. Differ. Equ. 2019, 311 (2019)

32. Bohner, M., Georgiev, S.G.: Multivariable Dynamic Calculus on Time Scales. Springer, Cham (2016)

33. Boukerrioua, K., Meziri, I., Chiheb, T.: Some refinements of certain Gamidov integral inequalities on time scales and applications. Kragujev. J. Math. 42(1), 131-152 (2018)

34. Li, W.N.: Some delay integral inequalities on time scales. Comput. Math. Appl. 59, 1929-1936 (2010)

35. Zheng, B., Zhang, Y.M., Feng, Q.: Some delay integral inequalities in two independent variables on time scales. J. Appl. Math. 1, Article ID 659563 (2011)

36. Li, W.N.: Some integral inequalities useful in the theory of certain dynamic equations on time scales. Comput. Math. Appl. 61, 1754-1759 (2011)

37. Li, W.N., Sheng, W.: Some Gronwall type inequalities on time scales. J. Math. Inequal. 4(1), 67-76 (2010) 